\title{
Multiple progressive intracranial arterial occlusions ('moyamoya' disease)
}

\author{
D. J . O’S U L L I V A N, G . H.K. L I M, P. D A R V E N I Z A, A N D \\ G. L. COF F E Y
}

From the Departments of Neurology and Radiology, St Vincent's Hospital, Sydney, Australia

SUMMARY Four adult female patients with moyamoya vessels are described. Hypertension and hyperlipidaemia were discovered in three patients. Vessels similar to moyamoya vessels were seen in the orbital and cortical collateral vessels as well as in the basal ganglia region. Attention should now be paid to the aetiological rather than the descriptive aspects of the disorder. An empirical trial of corticosteroids is worth while. Risk factors such as hypertension, hyperlipidaemia, and smoking should be eliminated.

In 1969 Suzuki and Takaku introduced the term cerebrovascular 'moyamoya' disease for a syndrome seen predominantly in young Japanese females, in which cerebral angiography showed an abnormal netlike appearance of small blood vessels at the base of the brain. Taveras (1969) reported 10 cases of this condition in children and young adults and used the term multiple progressive intracranial arterial occlusions.

The condition is characterised by stenosis or occlusion in the distal portions of the internal carotid artery, which may be accompanied by basilar artery occlusion or stenosis. Together with the occlusion there is the associated finding of a fine network of abnormal vessels ('moyamoya' vessels, that is 'puff of smoke' like appearance) in the region of the basal ganglia and arising from the trunks of the carotid, basilar, anterior, and middle cerebral arteries.

O'Sullivan (1973) reported a patient with this condition in Australia. Subsequently we have seen a further three patients and we believe they have hitherto undescribed features.

\section{Case reports}

\section{CASE 1}

This 22 year old married woman (VS) was admitted to hospital in November 1974 with a two month history of transient episodic dysarthria accompanied by weakness and numbness of the left

Address for reprint requests: Dr D. J. O'Sullivan, St Vincent's Hospital, Sydney, Australia.

Accepted 4 May 1977 arm and left leg. She was found to be hypertensive four years previously when pregnant. Each of her four pregnancies had been complicated by accelerated hypertension, toxaemia, prematurity and, in one instance, a miscarriage. Two years before admission she had an episode of weakness of the left arm and leg which improved with treatment of her hypertension. She smoked 10 cigarettes daily, and her father was both diabetic and hypertensive.

On examination there was some impairment of recent memory, dysarthria, and arteriovenous nipping in the fundi. There was a mild left hemiparesis and left hemianaesthesia. The deep tendon reflexes were increased on the left, abdominal reflexes were absent, and both plantar responses were flexor. The blood pressure was $250 /$ $150 \mathrm{mmHg}$ in both arms and there were no bruits over the carotid arteries, subclavian arteries or orbits. The remaining examination was normal.

Routine investigations were normal, including a normal fasting blood glucose, serum cholesterol of $4.9 \mathrm{mmol} / 1(190 \mathrm{mg} / \mathrm{dl})$, and serum triglyceride of $1.19 \mathrm{mmol} / 1(105 \mathrm{mg} / \mathrm{dl})$. A cerebral isotope encephalogram showed an area of increased uptake in the right parietal region and the electroencephalogram showed prominent right hemisphere delta wave activity.

\section{Angiography}

Bilateral carotid arteriography showed virtually symmetrical involvement of the carotid forks on both sides with extreme stenosis of the terminal portions of the internal carotid arteries and adjacent portions of the anterior and middle cerebral 


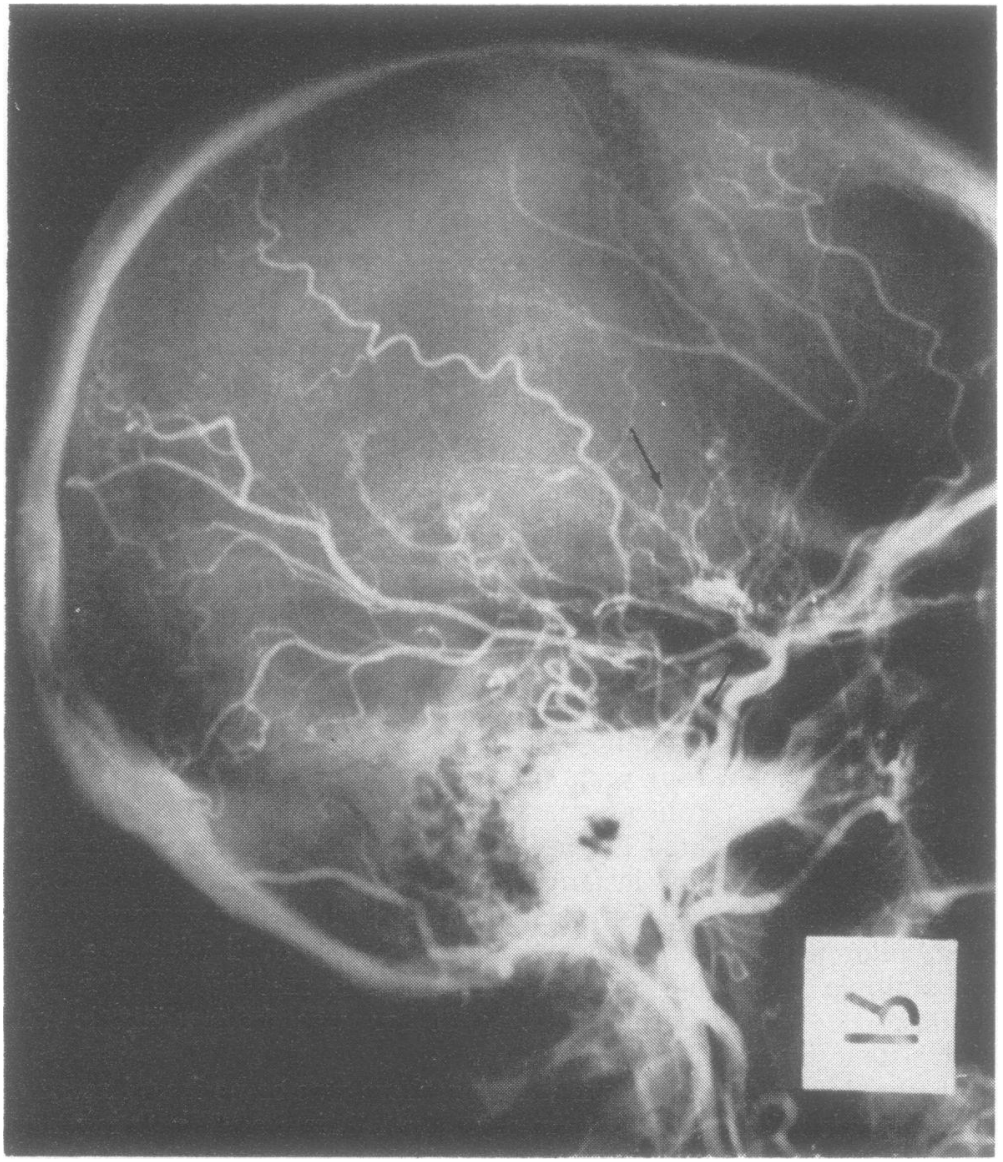

Fig. 1a Right carotid arteriography, lateral projection Stenosis of carotid fork and moyamoya vessels. arteries, and the appearance of networks of fine vessels in the region of the basal ganglia, consistent with moyamoya vessels (Figs. 1a,b). Vertebral arteriography showed marked narrowing of the terminal basilar artery and its superior cerebellar and posterior cerebral branches with tortuous and hypertrophied thalamo-perforate and posterior choroidal vessels, some of which anastomosed with the pericallosal artery serving as collateral to the impaired anterior cerebral circulation (Fig. 1c).

\section{Progress}

The patient improved on corticosteroids, dipyridamole, and control of her hypertension.

\section{CASE 2}

Mrs MS, aged 32 years with one child, presented in December 1968 with depression which did not respond to psychiatric therapy. Dementia and dys- arthria were soon noted. There was no relevant family history or past history.

Examination confirmed the presence of dementia, dysarthria, and emotional lability. She would not close her eyes to command, her tongue was spastic, and her jaw jerk increased. There were no vascular changes in the fundi. The remaining cranial nerves were normal. In the motor system there was a mild spastic quadriparesis, maximal in the left arm. All the deep tendon reflexes were abnormally brisk, abdominal reflexes were absent, and the plantar responses were flexor. There were no sensory or gross cerebellar deficits.

The blood pressure in both arms was $140 / 100$ $\mathrm{mmHg}$ and no bruits were heard over the head, orbits, or neck. Remaining examination was normal.

The serum cholesterol level was elevated at $8.0 \mathrm{mmol} / 1(310 \mathrm{mg} / \mathrm{dl})$, the serum triglycerides were $1.2 \mathrm{mmol} / \mathrm{l}(102 \mathrm{mg} / \mathrm{dl})$ and the lipid electro- 
phoretogram was normal. The electroencephalogram showed an excess of bitemporal slow wave activity.

\section{Angiography}

Arch aortography showed a normal aortic arch and its great branches. Bilateral common carotid arteriography showed reduced calibre of the internal carotid arteries with occlusions at the level of the siphons on both sides, just proximal to the ophthalmic arteries. The external carotid arteries were hypertrophied and gave rise to profuse intraorbital branches which anastomosed with the ophthalmic arteries, and, through the latter, supplied the anterior and middle cerebral arteries and the moyamoya vessels in the basal ganglia on both sides (Fig. 2). Vertebral arteriography demonstrated occlusion of the terminal portion of the basilar artery and hypertrophy of the posterior inferior (PICA) and anterior inferior (AICA) cerebellar arteries on both sides, whose branches supplied collateral flow to the superior cerebellar and posterior cerebral artery territories.

\section{Progress}

Despite treatment with low fat diet, hypotensive agents, and corticosteroids, this patient became bedridden and finally died in 1973 . Necropsy was not performed

\section{CASE 3}

Mrs IB, a 45 year old Indian female, first presented in August 1968 with an acute psychosis which settled quickly with electroconvulsive and psychotropic therapy. Neurological examination at this time was apparently normal.

She was readmitted in November 1972 with fluctuating weakness and numbness of the right arm and right leg for four weeks. She was on an oral contraceptive pill and smoked 20 cigarettes daily.

On examination there was impairment of recent

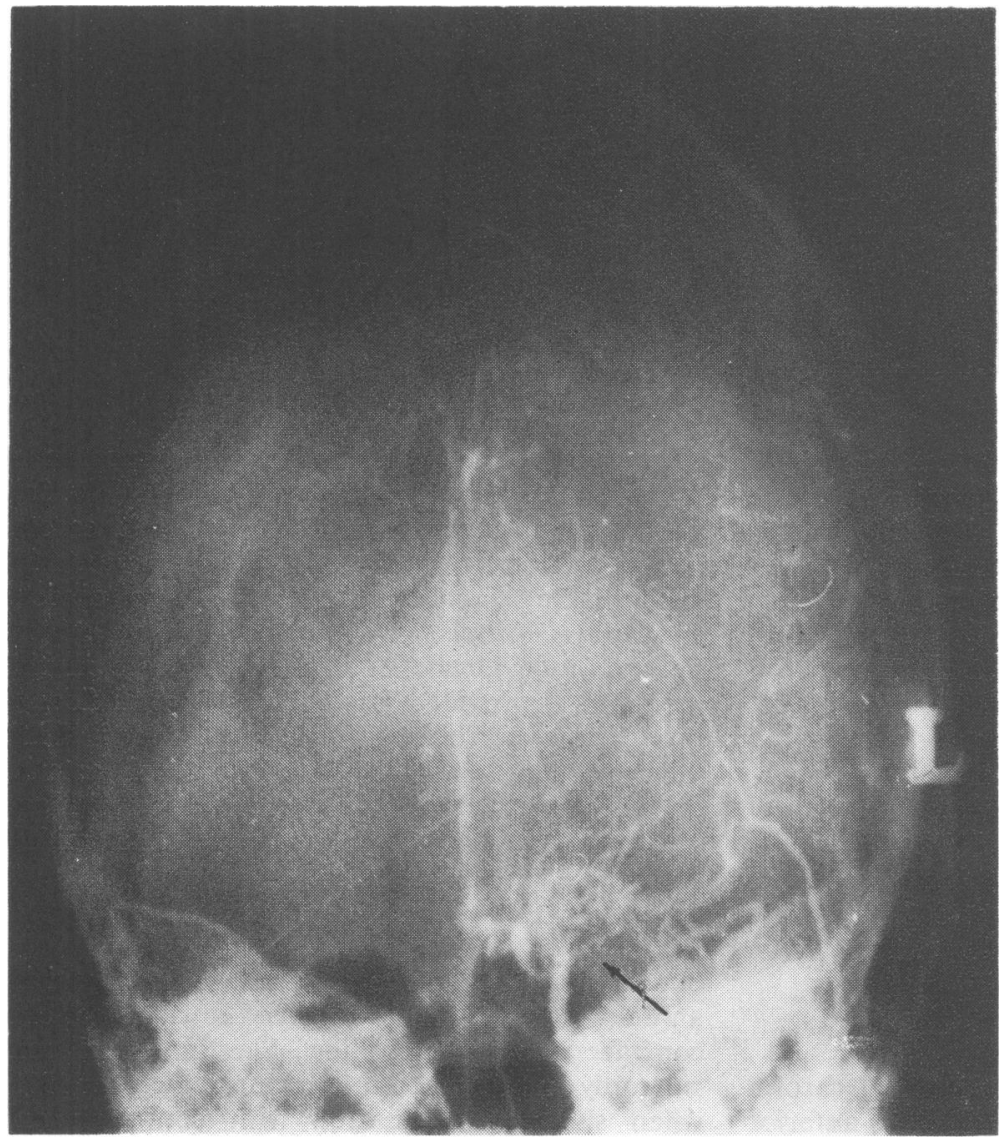

Fig. 1b Left carotid arteriography, $A-P$ projection. Stenosis of carotid fork and moyamoya vessels. 


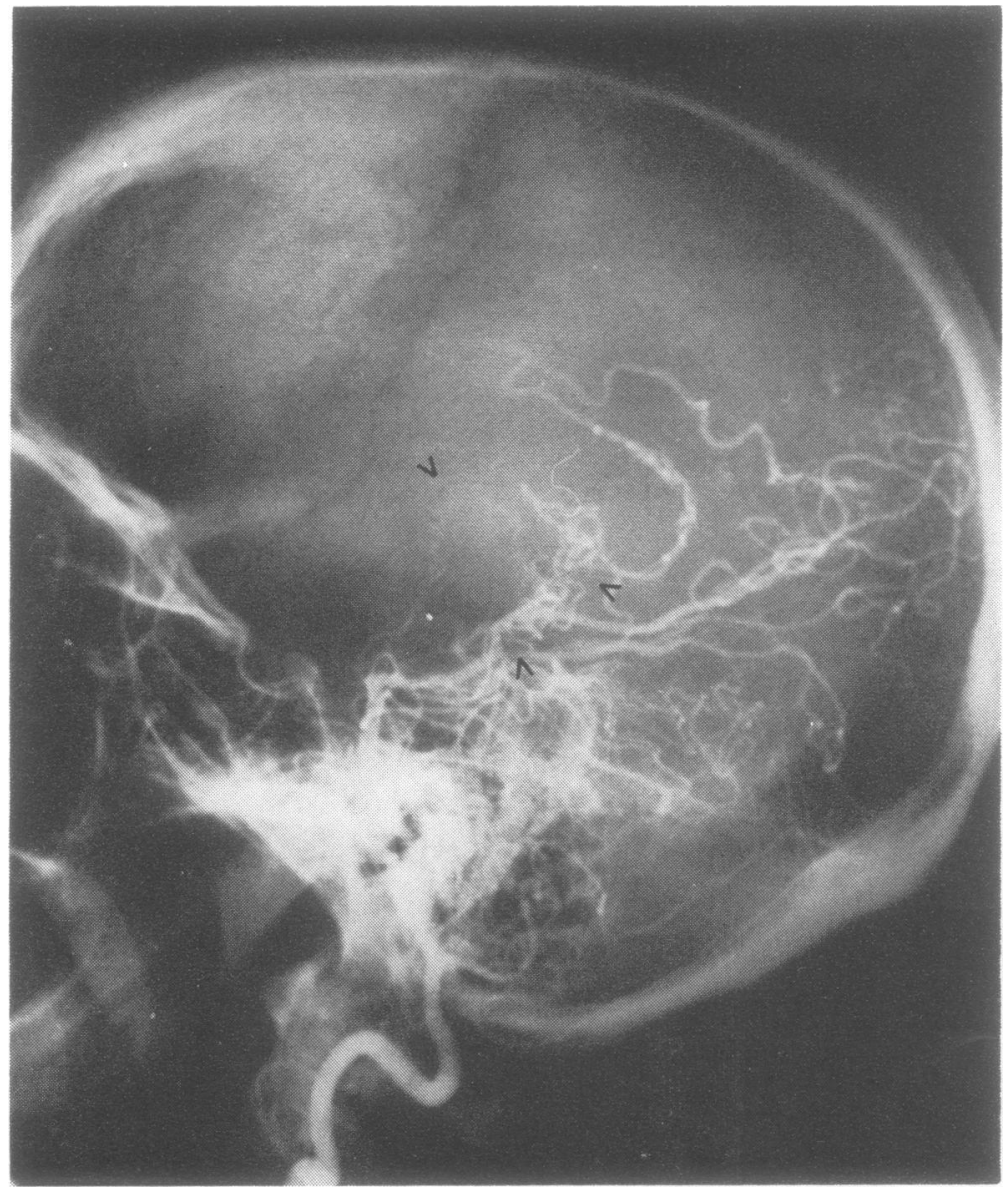

Fig. 1c Left vertebral arteriography, lateral projection. Hypertrophy of thalamoperforate and posterior choroidal vessels. memory. Cranial nerves were intact and there were no vascular changes in the fundi. There was a mild spastic hemiparesis of the right side accompanied by increased deep tendon reflexes. She was clumsy with her right arm and right leg. Sensory testing was normal.

The blood pressure was $120 / 180 \mathrm{mmHg}$ in both arms and a loud bruit was heard over the left orbit. Remaining examination was normal.

The serum cholesterol was $7.3 \mathrm{mmol} / 1(280 \mathrm{mg} /$ dl) and the serum triglycerides were $6.7 \mathrm{mmol} / 1$ $(590 \mathrm{mg} / \mathrm{dl})$. There was a type IV lipid electrophoretogram. The electroencephalogram showed excessive bilateral theta activity and the dynamic cerebral isotope scan showed reduced flow to the right hemisphere.

\section{Angiography}

There was occlusion of the right internal carotid artery at the level of the siphon and of its anterior and middle cerebral branches with appearances of moyamoya vessels in the basal ganglia (Fig. 3a). There was marked stenosis of the left carotid siphon and the adjacent portions of the anterior and middle cerebral arteries with no obvious moyamoya vessels. The vertebrobasilar system showed no evidence of occlusive disease in this case, but it provided important collateral flow to the carotid territories via the circle of Willis anteriorly and the posterior cerebral arteries posteriorly, whose branches anastomosed with and supplied branches of the anterior and middle cerebral arteries (Fig. 3b).

\section{Progress}

She was treated with low fat diet, soluble aspirin, and dipyridamole, and was asked to cease her oral contraceptive and smoking. She improved considerably and returned to work until October 1973, when she was readmitted with confusion and 
reoccurrence of her right hemiparesis. In addition, there was dementia, a grasp reflex with her right hand, and occasional dystonic movements in the left arm. Despite her diet, her lipid profile remained abnormal with a serum cholesterol of 8.3 $\mathrm{mmol} / 1(320 \mathrm{mg} / \mathrm{dl})$ and a serum triglyceride of $2.7 \mathrm{mmol} / 1(235 \mathrm{mg} / \mathrm{dl})$ and a type II lipid electrophoretogram. She again improved spontaneously, but remained demented.

\section{CASE 4}

Miss BS, aged 58 years, was admitted to hospital in August 1973 with a six week history of increasing weakness of the left arm and left leg. She had an episode of left sided weakness in February 1972 but this recovered gradually and she was subsequently well enough to go on a world tour. She had a previous history of pyelonephritis.

On examination there was dysarthria accompanied by a mild spastic quadriparesis greater on the left side. The deep tendon reflexes were all increased and the left plantar response was extensor. Sensation was intact and her gait ataxic. There were no vascular changes in the fundi. The blood pressure was $180 / 100 \mathrm{mmHg}$ in both arms and bruits were audible over the left carotid and both subclavian arteries. Remaining examination was normal.

Serum cholesterol was elevated at $7.5 \mathrm{mmol} / 1$ $(290 \mathrm{mg} / \mathrm{dl})$ and the serum triglycerides were 1.2 $\mathrm{mmol} / \mathrm{l}(105 \mathrm{mg} / \mathrm{dl})$. The lipid electrophoretogram was normal. The electroencephalogram showed excessive right hemisphere slow wave activity.

\section{Angiography}

There was complete occlusion of the internal carotid artery on each side at its upper end and reduced calibre of the main part of the artery. On each side there was good filling of the anterior choroidal artery and this appeared to feed back

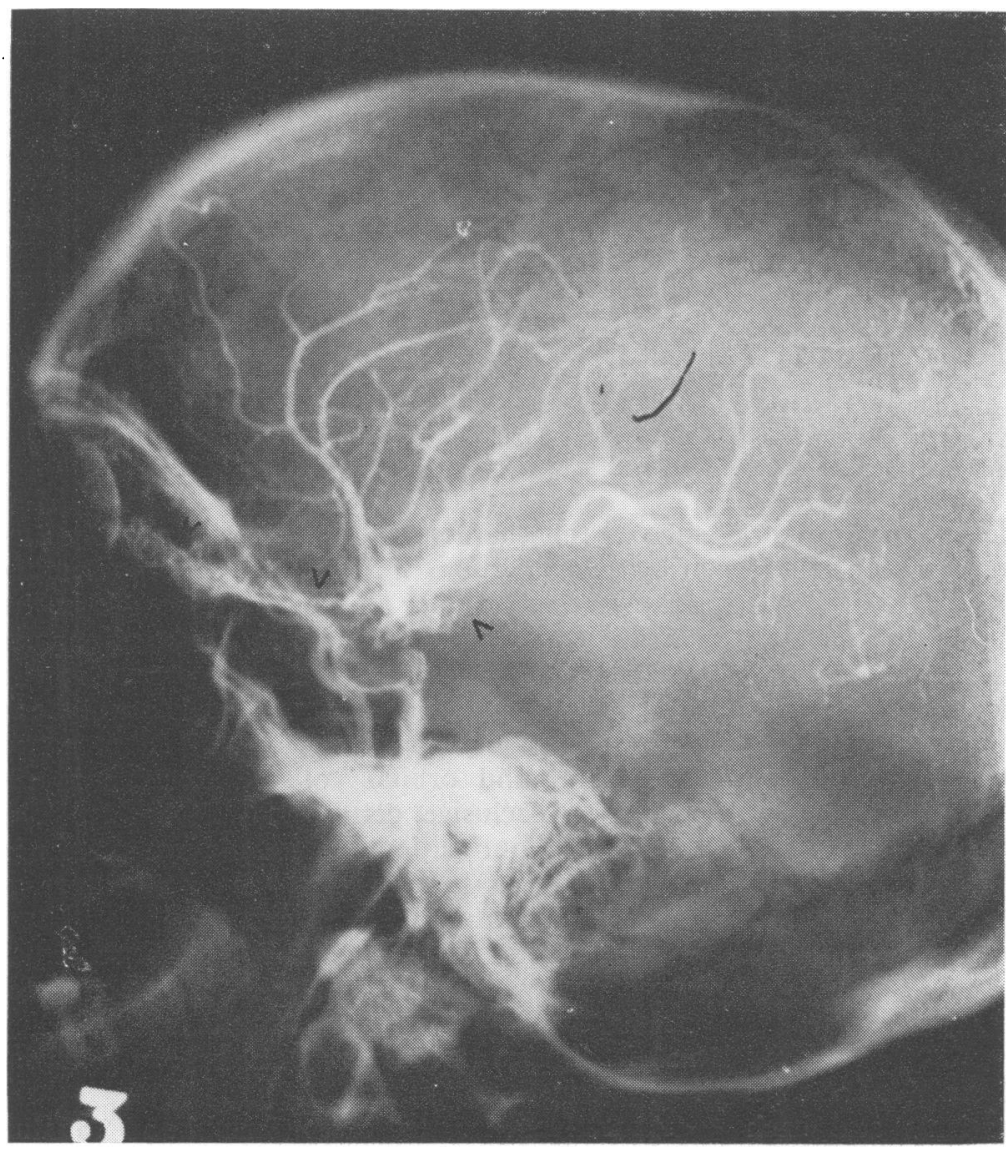

Fig. 2 Left carotid arteriography, lateral projection, showing luxuriant orbital anastomoses providing flow to ophthalmic and intracranial vessels. 


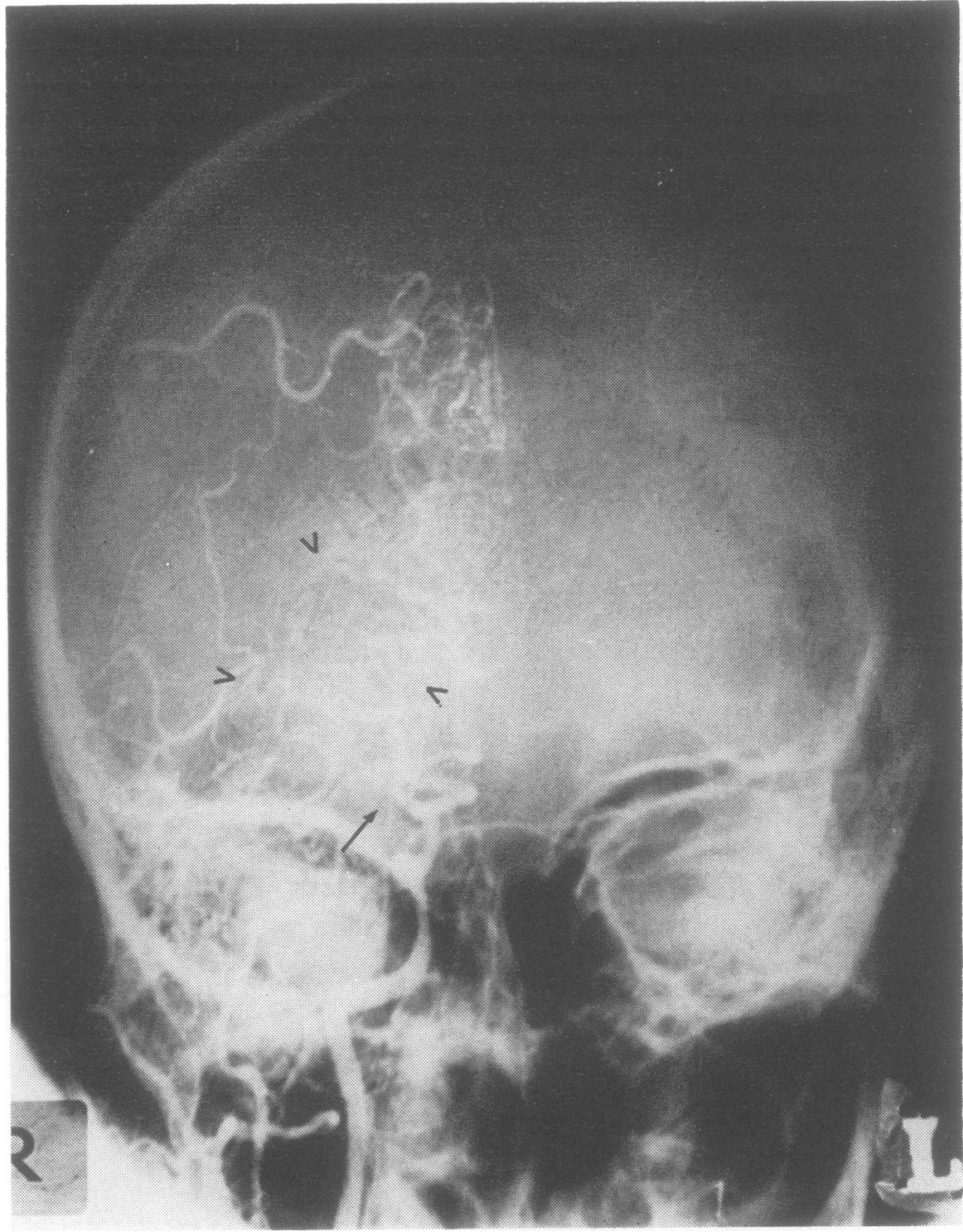

Fig. 3a Right carotid arteriography, A-P projection. Occlusion of carotid fork and moyamoya vessels. into an area of fine anastomotic channels in the thalamic region. No abnormality was seen in the vertebrobasilar system.

\section{Progress}

She was treated with a low fat diet, soluble aspirin, dipyridamole, and hypotensive agents with little improvement. She was readmitted in November 1973 with grand mal seizures, dementia, and increasing left hemiplegia. Anticonvulsants and corticosteroids were added to her treatment and she improved dramatically. She has continued to improve and remains independent.

\section{Discussion}

Angiography of the carotid and vertebral arteries was performed in all these patients and each showed the characteristic features previously described. In addition, three of the patients demonstrated vessels similar to moyamoya vessels in the cortical collaterals as well as in the basal ganglia region. Indeed in case 2 vessels are seen in the orbit of similar morphology. This has not been alluded to on angiography before. The appearance of cortical and orbital collateral vessels in response to occlusive disease is well known. The presence in our patients of moyamoya vessels in these situations supports their acquired nature as against congenital malformation.

All patients had abnormal electroencephalograms and two (cases 1 and 3 ) had abnormal cerebral isotope scans. This has been a common finding and it almost certainly reflects ischaemia or infarction in these patients.

Apart from isolated cases (Suzuki and Takaku, 


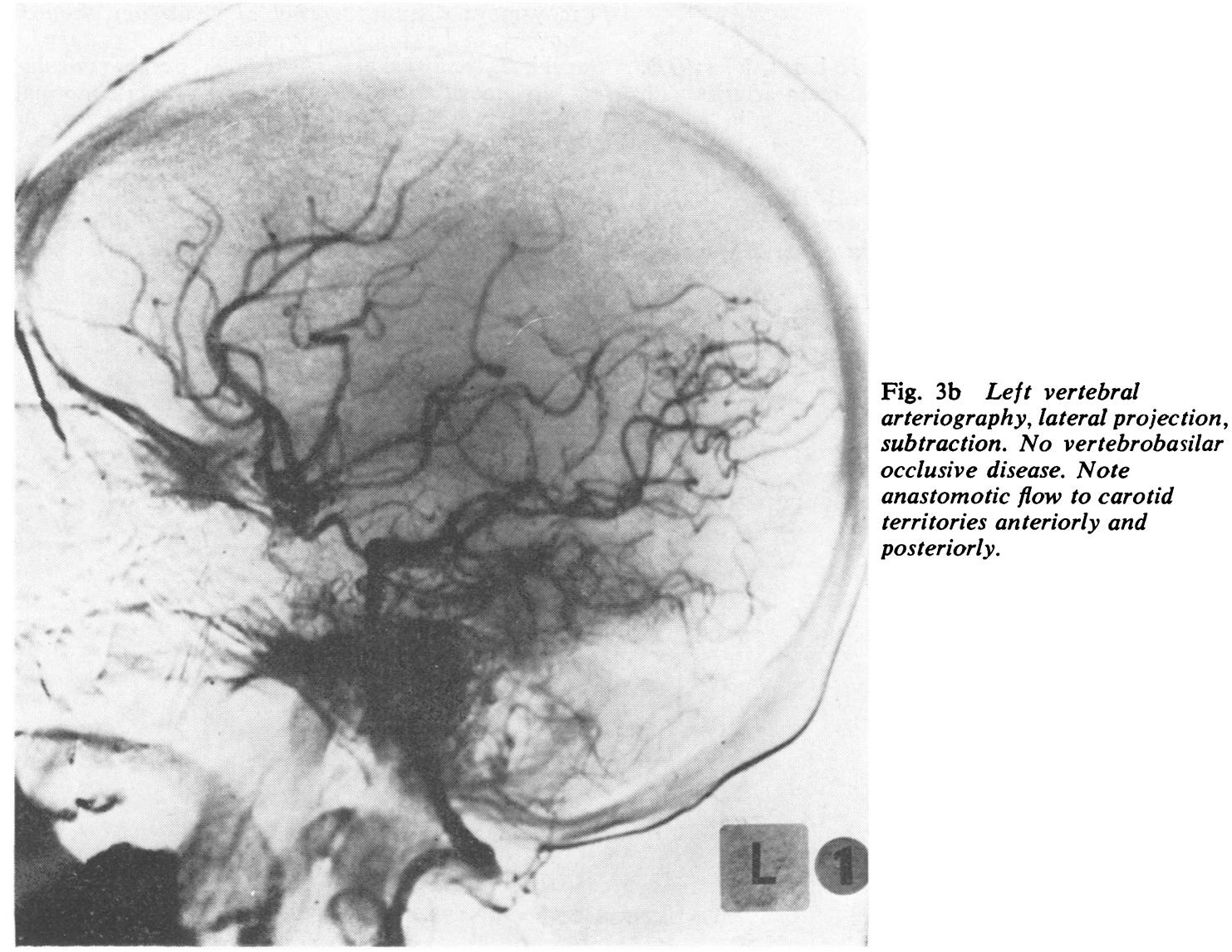

1969; Iivanainen et al., 1973 hyperlipidaemia has not been a major factor in patients with moyamoya disease. Hyperlipidaemia has been implicated in atherosclerotic cerebrovascular disease (Kannel, 1971). The findings of abnormal lipids in three of our four patients would tend to suggest an atherosclerotic aetiology.

Hyperlipidaemia should be looked for and treated in all patients with moyamoya vessels in the hope of preventing or delaying progress of the disease process. In addition, three of our patients (cases 1, 2, and 4) had hypertension which has emerged as a major risk factor in cerebrovascular disease (Kannel, 1971). Treatment of hypertension may well halt the occlusive process in these moyamoya patients and, furthermore, prevent intracranial haemorrhage which has been a common complication in adult patients. Cigarette smoking, another obvious risk factor should also be eliminated. Unfortunately, a necropsy could not be obtained in our patient who died (case 2). Poor and Gacs (1974) reported necropsies on two patients with moyamoya vessels who showed atherosclerotic occlusions of the cerebral vessels. It is the likely basis of the disease in our patients.

Three of our patients (cases 1, 2, and 4) were treated with corticosteroids. Two improved significantly. While it is well known that patients with this condition may improve or stabilise spontaneously, it would seem, in the light of our experience, that steroid therapy is worth a trial.

It is now becoming established in adult patients that moyamoya vessels are collaterals that develop in response to stenosis and occlusions of the distal carotid vessels of varying aetiology. Attention should now shift to a more detailed analysis of aetiological and pathological aspects in its rational management. 


\section{References}

Iivanainen, M., Vuolio, M., and Halonen, V. (1973). Occlusive disease of intracranial main arteries with collateral networks. Moyamoya disease in adults. Acta Neurologica Scandinavica, 49, 307-322.

Kannel, W. B. (1971). Risk factors in stroke due to cerebral infarction. Stroke, 2, 423-428.

O'Sullivan, D. J. (1973). Cerebrovascular "moyamoya" disease. Proceedings of the Australian Association of Neurologists, 9, 73-79.

Poor, G. Y., and Gacs, G. Y. (1974). The so-called moyamoya disease. Journal of Neurology, Neurosurgery, and Psychiatry, 37, 370-377.

Suzuki, J., and Takaku, A. (1969). Cerebrovascular "Moyamoya" disease. A disease showing abnormal netlike vessels in the base of the brain. Archives of Neurology (Chicago), 20, 288-299.

Taveras, J. M. (1969). Multiple progressive intracranial arterial occlusions. A syndrome of children and young adults. American Journal of Roentgenology, Radiation Therapy and Nuclear Medicine, 106, 235-268. 